\title{
AVALIAÇÃO DA GRANULOMETRIA DE ALIMENTAÇÃO E DOS PRODUTOS DA ETAPA ROUGHER DE FLOTAÇÃO DA USINA DO SOSSEGO
}

\author{
A. MIRANDA ${ }^{1}$, R. A. FONSECA ${ }^{1}$, F. OLEGARIO Jr. ${ }^{1}$, M. SOUZA ${ }^{1}$, G. OLIVEIRA ${ }^{1}$, \\ M. G. BERGERMAN ${ }^{2 *}$ e H. DELBONI Jr. ${ }^{2}$ \\ ${ }^{1}$ Vale S.A. \\ ${ }^{2}$ Universidade de São Paulo \\ mbergerman@usp.br*
}

Artigo submetido em novembro/2015 e aceito em novembro/2015

DOI: $10.15628 /$ holos.2015.3655

\section{RESUMO}

A usina do Sossego processa um minério sulfetado de cobre e ouro. A etapa de flotação compreende estágios rougher, cleaner e scavenger do cleaner, sendo que os concentrados das etapas rougher e scavenger do cleaner passam por um estágio de remoagem antes de seguir para a etapa cleaner. Ao longo dos primeiros anos de operação da usina, observou-se que a granulometria de alimentação da remoagem estava consistentemente mais fina que o esperado, mesmo considerando-se que a granulometria de alimentação da flotação (etapa rougher) estava dentro dos parâmetros esperados e significativamente mais grossa que o concentrado rougher. Este trabalho apresenta os resultados das amostragens de campo para identificar a origem de tal diferença e quantificar a mesma, assim como ilustra os impactos de tal diferença na etapa de remoagem do concentrado.

PALAVRAS-CHAVE: Flotação, Remoagem, Cobre, Classificação.

\section{EVALUATION OF THE ROUGHER FLOTATION FEED AND CONCENTRATE SIZE DISTRIBUITION AT THE SOSSEGO}

\begin{abstract}
Sossego plant processes a copper and gold sulfide ore. The flotation process comprises the rougher, cleaner and scavenger of cleaner stages. The concentrate from the rougher and scavenger of cleaner stages goes through a regrinding process before being fed into the cleaner stage. During the first years of operation, it was noticed that the regrinding feed size was consistently finer than expected, even though the flotation feed size (rougher
\end{abstract}

stage) was within the planned parameters and significantly coarser than the concentrate from the rougher stage. This paper describes the results of field samplings to identify the factors behind such difference in size and to quantify it. Moreover, it shows the impacts of such size difference on the concentrate regrinding stage.

KEYWORDS: Flotation, Regrinding, Copper, Classification. 


\section{INTRODUÇÃO}

A mina do Sossego, da Vale, localizada em Canaã dos Carajás, estado do Pará, iniciou suas operações em 2004, sendo a primeira mina de cobre da empresa. Esta possui uma reserva estimada de 245 milhões de toneladas com teores de 0,98\% de cobre e 0,28 g/t de ouro (para um cut off de $0,33 \%$ de cobre equivalente), divididos em dois corpos, denominados Sequeirinho (principal) e Sossego. Este último representa aproximadamente 15\% das reservas. Há também cerca de 16,5 milhões de toneladas de minério de cobre oxidado (VALE, 2001).

O minério de cobre é basicamente calcopíritico, com pequena presença de bornita e calcocita. A usina do Sossego processa 41.000 toneladas de minério de cobre por dia e está projetada para uma produção superior a 470.000 toneladas de concentrado de cobre por ano. 0 fluxograma de processo é apresentado na Figura 1.
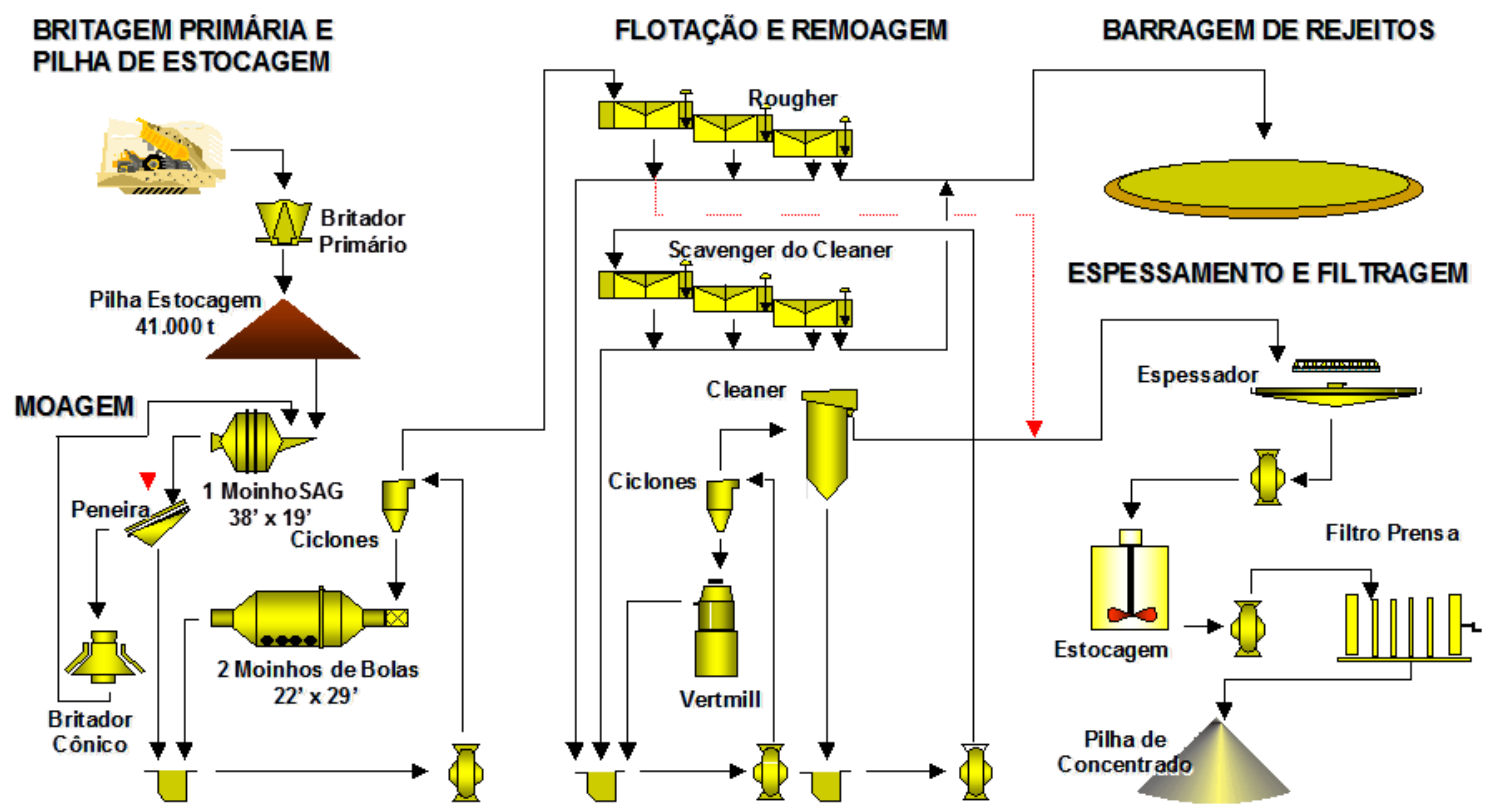

Figura 1. Fluxograma de processo da usina do Sossego (Fonte: ROSA et al.,2007)

O circuito da usina do Sossego é descrito em detalhes por Bergerman (2009) e Rosa et al. (2007). A seguir é apresentada uma breve descrição do circuito de flotação do Sossego, foco do presente trabalho. O overflow dos ciclones da moagem de bolas, com um $\mathrm{P}_{80}$ da ordem de $210 \mu \mathrm{m}$, segue para a etapa rougher de flotação. Os concentrados rougher e scavenger do cleaner da flotação alimentam, por meio de bombas de velocidade variável, duas baterias de ciclones. $O$ underflow da ciclonagem é direcionado, por gravidade, para a caixa de alimentação dos moinhos verticais, os quais são alimentados pela porção inferior do mesmo por meio de uma bomba que também opera com velocidade variável. Os dois moinhos verticais operam em circuito fechado com a ciclonagem. A descarga do moinho vertical passa por uma classificação interna, na qual o material grosseiro retorna diretamente ao moinho, em conjunto com a alimentação nova, e o material fino segue para a classificação em ciclones. 0 produto da remoagem (overflow dos ciclones), com $\mathrm{P}_{80}$ da ordem de $44 \mu \mathrm{m}$, é submetido à flotação cleaner em colunas de flotação, sendo o concentrado cleaner o produto final e o rejeito cleaner a alimentação da etapa scavenger do cleaner. 
Como medida para avaliar o desempenho da flotação, criou-se uma rotina de amostragens mensais do circuito completo de flotação. As amostras coletadas eram submetidas a análises granulométricas e química por faixa. Os resultados permitiam que a equipe de processo do Sossego pudesse sempre buscar otimizações nesta etapa do circuito. Uma avaliação dos dados quanto ao comportamento da distribuição granulométrica ao longo do circuito (Bergerman, 2013) mostrou que eram observadas significativas diferenças de granulometria entre a alimentação da flotação rougher e o concentrado da mesma etapa. Tal diferença não foi prevista em projeto, tendo em vista que a granulometria esperada para a alimentação dos moinhos verticais proveniente do concentrado rougher foi determinada como a mesma granulometria da alimentação da etapa rougher. O presente trabalho tem por objetivo quantificar tal diferença e avaliar o impacto da mesma sobre a flotação e remoagem da usina do Sossego.

\section{METODOLOGIA}

Para a etapa de diagnóstico do circuito, foram coletadas amostras nos seguintes pontos da usina do Sossego:

- Overflow dos ciclones da moagem de bolas (alimentação da flotação rougher);

- Concentrado rougher;

- Rejeito rougher;

- Alimentação cleaner;

- Concentrado cleaner;

- Rejeito cleaner (alimentação da flotação scavenger do cleaner);

- Concentrado scavenger do cleaner;

- Rejeito scavenger do cleaner.

- Rejeito final (tubulação que reúne o rejeito rougher e o rejeito scavenger do cleaner).

A Figura 2 ilustra o amostrador utilizado para a tomada das amostras da ciclonagem e a Tabela 1 ilustra os fatores utilizados no cálculo da massa mínima a ser coletada em cada fluxo.

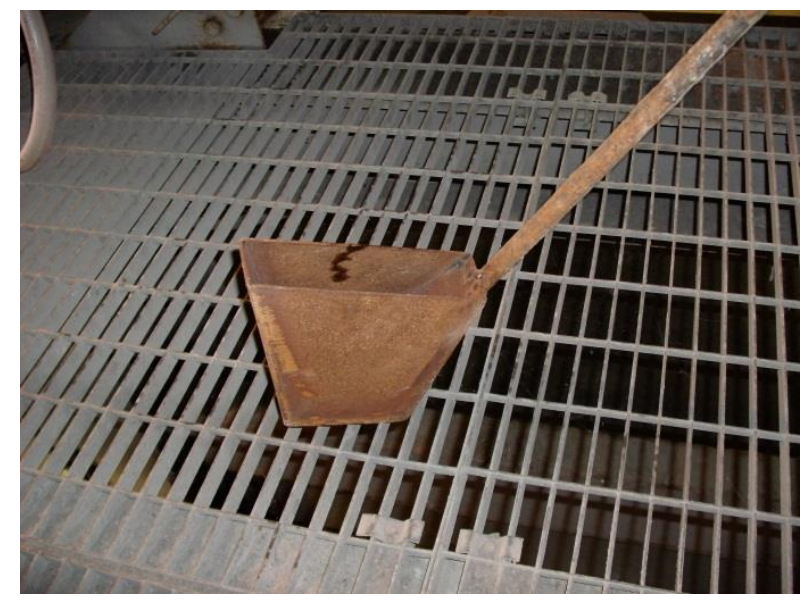

Figura 2 - Amostrador utilizado para amostragem da ciclonagem 
Tabela 1 - Cálculo das massas mínimas utilizando a fórmula proposta por Gy (1992).

\begin{tabular}{|c|c|c|c|c|c|c|c|c|}
\hline Fluxo & $\begin{array}{l}\text { Fator de } \\
\text { forma } \\
\text { do } \\
\text { material }\end{array}$ & $\begin{array}{l}\text { Densidade } \\
\text { do } \\
\text { material } \\
\left(\mathrm{g} / \mathrm{cm}^{3}\right)\end{array}$ & $\begin{array}{l}\text { Tamanho } \\
\text { máximo } \\
\text { do } \\
\text { intervalo } \\
\text { de } \\
\text { interesse } \\
\text { (mm) }\end{array}$ & $\begin{array}{l}\text { Nível de } \\
\text { confiança } \\
(\%)\end{array}$ & $\begin{array}{l}\text { Precisão } \\
(\%)\end{array}$ & $\begin{array}{l}\text { Proporção } \\
\text { esperada } \\
\text { no } \\
\text { intervalo } \\
\text { de } \\
\text { interesse }\end{array}$ & $\begin{array}{l}\text { Ordenada } \\
\text { normal } \\
\text { no nível } \\
\text { de } \\
\text { confiança } \\
\text { escolhido }\end{array}$ & $\begin{array}{c}\text { Massa } \\
\text { mínima } \\
\text { (g) }\end{array}$ \\
\hline $\begin{array}{l}\text { Alimentação } \\
\text { rougher }\end{array}$ & 0,60 & 3,62 & 0,05 & 95 & 10 & 20 & 1,96 & 260 \\
\hline $\begin{array}{l}\text { Concentrado e } \\
\text { rejeito rougher }\end{array}$ & 0,60 & 3,62 & 0,3 & 99,9 & 10 & 35 & 3,29 & 93 \\
\hline $\begin{array}{l}\text { Concentrado e } \\
\text { rejeito scavenger } \\
\text { e Concentrado e } \\
\text { rejeito cleaner }\end{array}$ & 0,60 & 4,00 & 0,3 & 99,9 & 10 & 35 & 3,29 & 104 \\
\hline $\begin{array}{l}\text { Alimentação } \\
\text { cleaner }\end{array}$ & 0,60 & 3,62 & 0,3 & 99,9 & 10 & 35 & 3,29 & 93 \\
\hline
\end{tabular}

As amostragens foram sempre realizadas durante um período de duas horas, no qual a usina foi considerada sob operação estável, com incrementos tomados a cada 15 minutos. Um ponto importante de atenção durante a amostragem foi quanto à operação de bombas de poço, que caso em operação, podem desestabilizar o circuito. No presente trabalho, adotou-se o procedimento de não operar as mesmas $2 \mathrm{~h}$ antes da realização das amostragens.

As amostras foram enviadas para o laboratório de processo da usina do Sossego. Inicialmente, foram determinadas as porcentagens de sólidos de todas as amostras. Em seguida, foi realizado o peneiramento a úmido, com telas nas seguintes aberturas: 425, 212, 150, 106, 75, 45 e $38 \mu \mathrm{m}$. O produto retido em cada malha foi encaminhado para análise química no Laboratório Químico da usina do Sossego, para determinação do teor de cobre por meio de absorção atômica.

Para a realização deste trabalho, foram realizadas 38 amostragens entre os anos de 2008 e 2012.

\section{RESULTADOS E DISCUSSÕES}

A partir dos resultados das amostragens no circuito industrial, foram reunidos dados da granulometria de alimentação da flotação rougher e comparados com os dados da granulometria do concentrado rougher, que constitui a alimentação nova do circuito de remoagem. Estes dados estão ilustrados na Figura 3. 


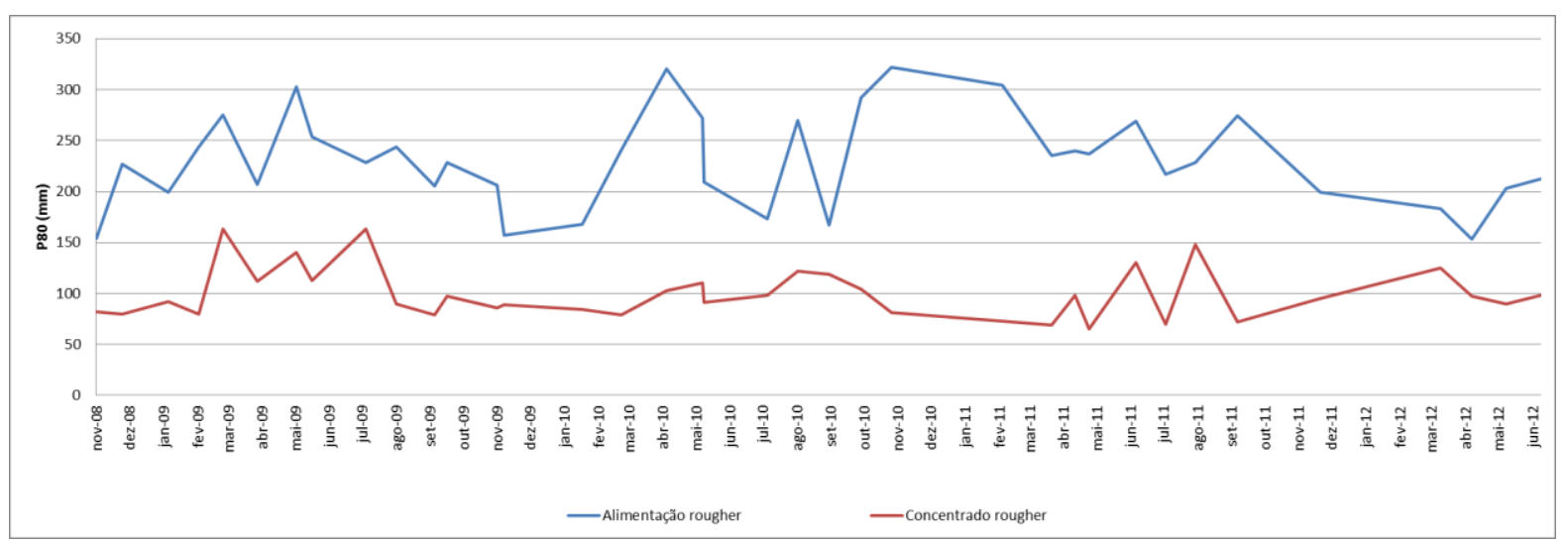

Figura 3 - Comparação da granulometria de alimentação rougher e do concentrado rougher

Os dados da Figura 3 ilustram uma clara tendência de uma granulometria significativamente mais fina do concentrado rougher em relação à alimentação da mesma etapa. Essa diferença se deve possivelmente a dois fatores. O primeiro é que partículas mais grosseiras podem não estar liberadas ou são pesadas demais para serem flotadas, sendo encaminhadas assim para o rejeito. $O$ segundo é que os sulfetos presentes no minério do Sossego, que são concentrados na etapa rougher, apresentam densidade significativamente maior que o restante da ganga, constituída principalmente de óxidos e silicatos. Essa diferença de densidade provavelmente faz com que partículas de sulfetos de mesmo tamanho das partículas de ganga sejam encaminhadas para o underflow do ciclone da moagem de bolas, sendo assim moídas além do necessário. Tal diferença possui um significativo impacto positivo sobre a operação dos moinhos verticais da usina do Sossego, que recebem uma alimentação com granulometria aproximadamente $50 \%$ mais fina que a projetada.

A fim de avaliar o impacto na recuperação da etapa rougher desta diferença entre as granulometrias da alimentação e do concentrado, foi determinada a recuperação por faixa granulométrica para todos os resultados de amostragens. Os resultados dos valores médios para as amostragens realizadas estão ilustrados na Tabela 2 .

Tabela 2 - Recuperação metalúrgica de cobre por faixa granulométrica - média das amostragens de 2008 a 2011

\begin{tabular}{ccc}
\hline Malha $(\mathbf{m m})$ & Recuperação metalúrgica de cobre (\%) & Partição do cobre por fração (\%) \\
\hline 425 & 7,1 & 1,2 \\
212 & 67,8 & 8,0 \\
150 & 86,5 & 5,9 \\
106 & 93,1 & 7,9 \\
75 & 95,1 & 10,5 \\
45 & 96,2 & 11,2 \\
38 & 95,2 & 5,1 \\
$<38$ & 95,0 & 50,3 \\
Média etapa & 92,9 & 100 \\
\hline
\end{tabular}

Pode-se observar que existe uma queda significativa da recuperação de cobre nas frações mais grosseiras. Esta menor recuperação não impacta de forma significativa a recuperação média da etapa, tendo em vista que a quantidade de cobre presente nas frações mais grosseiras é muito baixa, de aproximadamente $10 \%$ nas frações acima de $212 \mu \mathrm{m}$, como também pode ser visto na Tabela 2. Como informação, a etapa rougher representa de 85 a $95 \%$ do rejeito final da usina, conforme resultados das amostragens industriais realizadas para o presente estudo. 
Nas etapas cleaner e scavenger do cleaner, não se observou o mesmo comportamento da etapa rougher - as granulometrias das alimentações e dos concentrados possuem distribuições granulométricas semelhantes.

\section{CONCLUSÃO}

O presente trabalho mostrou que existe uma significativa diferença entre a distribuição granulométrica da alimentação e do concentrado da etapa rougher de flotação da usina do Sossego. Uma significativa parcela da porção mais grosseira que alimenta a etapa rougher do circuito de flotação segue diretamente para o rejeito da etapa. Tal situação não impacta de forma significativa a recuperação metalúrgica do processo, tendo em vista que apenas aproximadamente $10 \%$ do cobre contido na alimentação da flotação se encontra nas frações mais grosseiras. Observa-se, no entanto, um impacto positivo significativo sobre a operação dos moinhos verticais, que recebem uma alimentação muito mais fina que a planejada em projeto. Tal característica pode ocorrer em outras usinas que processem minerais sulfetados e deve ser levada em conta no dimensionamento dos moinhos verticais.

\section{REFERÊNCIAS BIBLIOGRÁFICAS}

1. BERGERMAN, M.G. Modelagem e simulação do circuito de moagem do Sossego. 2009. 207 p. Dissertação (Mestrado) - Escola Politécnica, Universidade de São Paulo, São Paulo, 2009.

2. BERGERMAN, M.G. Dimensionamento e simulação de moinhos verticais. 2013. 282 p. Tese (Doutorado) - Escola Politécnica, Universidade de São Paulo, São Paulo, 2013.

3. GY, P. Sampling of heterogeneous and dynamic material systems: theories of heterogeneity, sampling and homogenizing. Amsterdam: Elsevier, 1992. 653 p.

4. ROSA, M.A.N., BERGERMAN, M.G., MIRANDA, A., OLIVEIRA, J.L., SOUZA, M., BATISTA FILHO, J., CARDOSO, W. Controle operacional da usina do Sossego. In: Meeting of the southern hemisphere on mineral technology e Encontro nacional de tratamento de minérios e metalurgia extrativa. Proceedings do VII Meeting of the southern hemisphere on mineral technology e XXII Encontro nacional de tratamento de minérios e metalurgia extrativa. Ouro Preto: Universidade Federal de Ouro Preto, 2007. v. III. p. 505-512.

5. VALE. Relatório de viabilidade do Projeto Sossego. Carajás: Minerconsult, Bechtel, ECM e Concremat, 2001. 451 p. (Relatório interno). 